\title{
Merrifield-Simmons index and its entropy of the 4-8-8 lattice
}

\author{
Zuhe Zhang \\ Department of Mathematics and Statistics \\ The University of Melbourne \\ Parkville, VIC 3010, Australia \\ zhang.zuhe@gmail.com
}

\begin{abstract}
The Merrifield-Simmons index is a topological index used in structural chemistry for quantifying relevant properties of molecular structure. This index, in different names, has been studied on the square, triangular and hexagonal lattices by using the method of transfer matrix. To compute the Merrifield-Simmons index of more complicated lattices such as the 4-8-8 and triangular kagomé lattices, the use of multi-step transfer matrices is needed. In this paper we introduce the concept of transfer multiplicity and use multi-step transfer matrices to derive a rigorous upper and lower bound for the entropy of Merrified-Simmons index of the 4-8-8 lattice. We show that this method can be used to deal with other complex 2-dimensional lattices.
\end{abstract}

\section{Introduction}

In the study of structural chemistry, Merrifield and Simmons defined a topological space for the chemical graphs [15]; the cardinality of this topological space is called the Merrified-Simmons index. In the context of combinatorics, for a graph $G$, the Merrifield-Simons index is the number of vertex independent sets of $G$. The properties of Merrifield-Simmons index of some types of benzenoids and polyominoes have been studied in $[8,22,24,25]$. In statistical mechanics, an essentially same problem is to count the ways of putting particles in the sites of a 2-dimensional lattice such that no two share the same site or are in adjacent sites $[2-4,6,7,16]$.

The 4-8-8 lattice is an Archimedean tilling which has been used to describe phase transitions in the layered hydrogen-boded $\mathrm{SnCL}^{2} \cdot 2 \mathrm{H}_{2} \mathrm{O}$ crystal [18] in physical systems. The triangular kagomé lattice corresponds to the positions of $\mathrm{Cu}$ atoms in the fabricated materials $\mathrm{Cu}_{9} \mathrm{X}_{2}(\mathrm{cpa})_{6} \cdot \mathrm{xH}_{2} \mathrm{O}(\mathrm{cpa}=2$-carboxypentonic acid, an derivative of asorbic acid; $\mathrm{X}-\mathrm{F}, \mathrm{Cl}, \mathrm{Br})$ [14]. Various physical models such as the spanning trees, dimer covering and bond percolation have been studied on these two lattices $[1,9,12,13,18-21]$. To compute the Merrifield-Simmons index and its entropy of such complicated lattices, using single-step transfer matrix as in [5] is not compatible. In this paper, we propose the concept of transfer multiplicity and use multi-step transfer matrices to overcome such limitation. We demonstrate our method on the 4-8-8 lattice, provide numerical results of the Merrified-Simmons index and a rigorous bound of its entropy. 


\section{Transfer matrix}

Let $G_{m, n}$ (where $m$ and $n$ always denote positive integers) denote a finite section of the 4-8-8 lattice whose hexagons are arranged in $m$ rows and $n$ columns as shown in Fig.1.

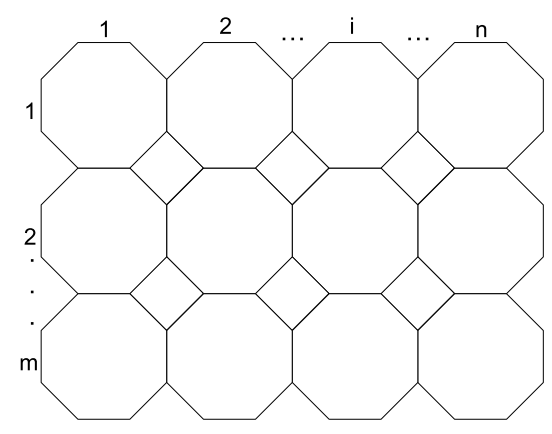

Fig. 1: $G_{m, n}$

Given an independent set $S$ of $G_{m, n}$, a portion of $S$ that lies in a fixed column of $G_{m, n}$ can be represented by either an $(m+1)$-vector or a $2 m$-vector of 0 's and 1's, where a 1 indicates that the vertex is in $S$ and a 0 indicates that the vertex is not in $S$. Thus, any independent set of $G_{m, n}$ can be represented by $3 n+1$ column vectors.

Example 2.1. Fig.2 below shows an independent set $\mathrm{S}$ in $G_{3,4}$. The portions of $\mathrm{S}$ that lie in each of the columns are represented by the respective vectors $(1,0,0,0,1,0),(0,1,0,1),(1,0,1,0)$, $(0,0,1,0,0,1),(0,0,1,0),(0,1,0,1),(1,0,0,1,1,0),(0,0,0,0),(1,1,1,1),(0,0,0,0,0,0),(0,1,0,0)$, $(0,0,1,1),(0,1,1,0,0,0)$.

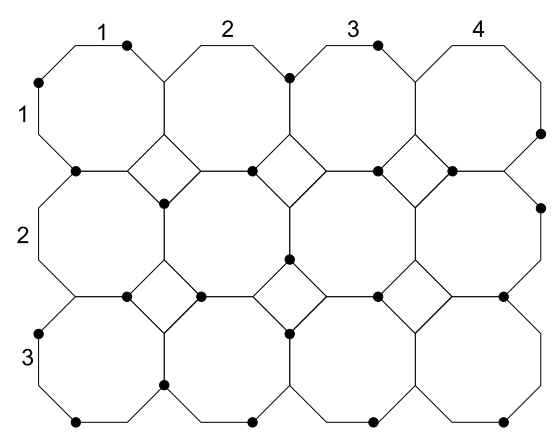

Fig. 2: An independent set of $G_{3,4}$.

Let $\mathbf{P}_{m}$ denote the set of all possible $(m+1)$-vectors which may represent a column in an independent set of $G_{m, n}$. The $(3 k+2)$-th and $(3 k+3)$-th columns of an independent set of $G_{m, n}$ are chosen from $\mathbf{P}_{m}$, where $0 \leq k \leq n-1$. Clearly, $\mathbf{P}_{m}$ is a collection of $(m+1)$-vectors of 0's and 1's, and the cardinality of $\mathbf{P}_{m}$ is $2^{m+1}$. Similarly, let $\mathbf{Q}_{m}$ denote the set of all possible $2 m$-vectors which may represent a $(3 k+1)$-th column in an independent set of $G_{m, n}$, where $0 \leq k \leq n$. It's easy to see that $\mathbf{Q}_{m}$ consists of $2 m$-vectors of 0's and 1's in which no consecutive 1 's occupy the positions of the $(2 k-1)$-th and $2 k$-th entries, for $1 \leq k \leq m$. Since there are 
three possibilities at each pair of consecutive $(2 k-1)$-th and $2 k$-th positions, the set $Q_{m}$ has $3^{m}$ vectors.

Let $\mathbf{v}(i)$ denote the $i$-th element of a vector $\mathbf{v}$. Note that two column vectors $\mathbf{v}, \mathbf{v}^{\prime}$ need to fit into one of the following two cases to be a possible consecutive pair of columns in an independent set of $G_{m, n}$,

(i) $\mathbf{v}$ and $\mathbf{v}^{\prime}$ are chosen from $\mathbf{P}_{m}$ and they are orthogonal to each other.

(ii) $\mathbf{v}, \mathbf{v}^{\prime}$ are chosen from $\mathbf{P}_{m}$ and $\mathbf{Q}_{m}$ respectively and they satisfy the following conditions

(a) $\mathbf{v}(1) \cdot \mathbf{v}^{\prime}(1)=0$

(b) $\mathbf{v}(i) \cdot \mathbf{v}^{\prime}(2 i-2)=0$ and $\mathbf{v}(i) \cdot \mathbf{v}^{\prime}(2 i-1)=0$ for $i=2,3, \ldots, m$,

(c) $\mathbf{v}(m+1) \cdot \mathbf{v}^{\prime}(2 m)=0$.

For fixed $m$ and $n$, all possible independent sets $S$ of $G_{m, n}$ can be obtained by a gluing process described below. First, take a vector from $\mathbf{Q}_{m}$ such that it corresponds to the first column of $S$, and denote it as $\mathbf{u}$. Second, to the right of $\mathbf{u}$ we glue a vector $\mathbf{v}$ selected from $\mathbf{P}_{m}$, making sure that $\mathbf{u}$ and $\mathbf{v}$ satisfy (ii). Third, we glue a vector $\mathbf{v}^{\prime}$ from $\mathbf{P}_{m}$ to the right of $\mathbf{v}$ under the condition that $\mathbf{v}$ and $\mathbf{v}^{\prime}$ satisfy (i). Then, we glue $\mathbf{u}^{\prime}$ to the right of $\mathbf{v}^{\prime}$ such that $\mathbf{v}^{\prime}$ and $\mathbf{u}^{\prime}$ satisfy (ii). Repeat the above procedure until the $(3 n+1)$-th column is glued.

Let us call the gluing of the $(i+1)$-th column $v_{i+1}$ to the right of $i$-th column $v_{i}$ as step $i$. Thus the transfer matrix representing step one, denoted $T_{m_{1}}=\left(T_{v_{1}, v_{2}}\right)$, is a $3^{m} \times 2^{m+1}$ matrix whose rows are indexed by vectors of $Q_{m}$ and columns are indexed by vectors of $P_{m}$, where $T_{v_{1}, v_{2}}=1$ if $v_{1}$ and $v_{2}$ represent possible consecutive pair of columns in an independent set of $G_{m, n}$ and $T_{v_{1}, v_{2}}=0$ otherwise. Note that the matrix depends only on $n$.

Similarly, the transfer matrix for step two is a $2^{m+1} \times 2^{m+1}$ matrix $T_{m_{2}}$ whose rows and columns are indexed by vectors of $P_{m} . T_{m_{2}}$ 's entry in position $(\alpha, \beta)$ is 1 if the vectors represented by $\alpha, \beta$ are orthogonal, and is 0 otherwise. The transfer matrix, $T_{m_{3}}$, for step three is the transpose of $T_{m_{1}}$. Note that $T_{m_{1}}$ is the transfer matrix for every step $i$ when $i=3 k+1$ $(0 \leq k \leq n-1), T_{m_{2}}$ is the transfer matrix for every step $i$ when $i=3 k+2(0 \leq k \leq n-1)$ and $T_{m_{3}}$ is the transfer matrix for every step $i$ when $i=3 k+3(0 \leq k \leq n-1)$. Thus, if we take the transfer matrix of $G_{m, n}$ to be $T_{m}=T_{m_{1}} T_{m_{2}} T_{m_{3}}$, then it is well known that for the Merrified-Simmons index of $G_{m, n}$, we have

$$
f(m, n)=\mathbf{1} \cdot T_{m}^{n} \mathbf{1}
$$

where 1 denotes the all one column vector. We call $T_{m}$ a triple-step transfer matrix since it is given as the product of three transfer matrices.

When $m=2$, the transfer matrices $T_{2_{1}}, T_{2_{2}}$ and $T_{2_{3}}$ are: 


$$
T_{2_{1}}=\left(\begin{array}{llllllll}
1 & 1 & 1 & 1 & 1 & 1 & 1 & 1 \\
1 & 0 & 1 & 0 & 1 & 0 & 1 & 0 \\
1 & 1 & 0 & 0 & 1 & 1 & 0 & 0 \\
1 & 1 & 0 & 0 & 1 & 1 & 0 & 0 \\
1 & 0 & 0 & 0 & 1 & 0 & 0 & 0 \\
1 & 1 & 0 & 0 & 1 & 1 & 0 & 0 \\
1 & 1 & 1 & 1 & 0 & 0 & 0 & 0 \\
1 & 0 & 1 & 0 & 0 & 0 & 0 & 0 \\
1 & 1 & 0 & 0 & 0 & 0 & 0 & 0
\end{array}\right), T_{2_{2}}=\left(\begin{array}{cccccccc}
1 & 1 & 1 & 1 & 1 & 1 & 1 & 1 \\
1 & 0 & 1 & 0 & 1 & 0 & 1 & 0 \\
1 & 1 & 0 & 0 & 1 & 1 & 0 & 0 \\
1 & 0 & 0 & 0 & 1 & 0 & 0 & 0 \\
1 & 1 & 1 & 1 & 0 & 0 & 0 & 0 \\
1 & 0 & 1 & 0 & 0 & 0 & 0 & 0 \\
1 & 1 & 0 & 0 & 0 & 0 & 0 & 0 \\
1 & 0 & 0 & 0 & 0 & 0 & 0 & 0
\end{array}\right), T_{2_{3}}=T_{2_{1}}^{\top}
$$

Thus, the triple-step transfer matrix of $G_{m, n}$ is:

$$
T_{2}=\left(\begin{array}{ccccccccc}
27 & 18 & 18 & 18 & 12 & 18 & 18 & 12 & 12 \\
18 & 9 & 12 & 12 & 6 & 12 & 12 & 6 & 8 \\
18 & 12 & 9 & 9 & 6 & 9 & 12 & 8 & 6 \\
18 & 12 & 9 & 9 & 6 & 9 & 12 & 8 & 6 \\
12 & 6 & 6 & 6 & 3 & 6 & 8 & 4 & 4 \\
18 & 12 & 9 & 9 & 6 & 9 & 12 & 8 & 6 \\
18 & 12 & 12 & 12 & 8 & 12 & 9 & 6 & 6 \\
12 & 6 & 8 & 8 & 4 & 8 & 6 & 3 & 4 \\
12 & 8 & 6 & 6 & 4 & 6 & 6 & 4 & 3
\end{array}\right)
$$

By identifying edges $\left(a_{i}, a_{i+1}\right)$ with $\left(a_{i}^{*}, a_{i+1}^{*}\right)(1 \leq i \leq 2 m-1)$ of $G_{m, n}$ respectively, the 4-8-8 lattice with cylindrical boundary condition, denote as $H_{m, n}$, can be obtained. Note that here the graph can be seen as drawn on a vertical cylinder.

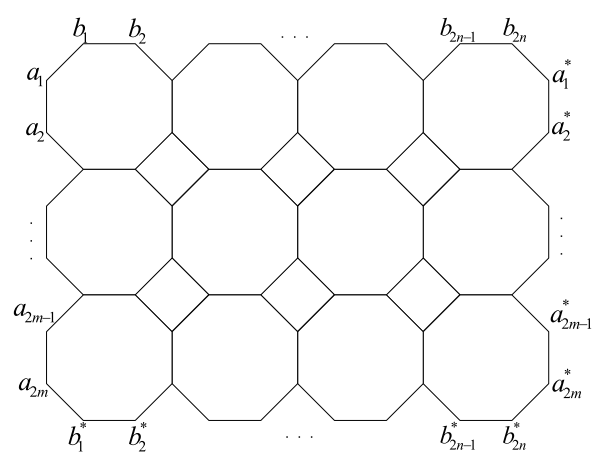

Fig. 3: The 4-8-8 lattice $G_{m, n}$ with free boundary condition

Similar to the discussion of $G_{m, n}$, any independent set $I$ of $H_{m, n}$ can be generated by gluing vectors from $\mathbf{P}_{n-1}$ and $\mathbf{Q}_{n}$. Note that if $\mathbf{v}$ and $\mathbf{v}^{\prime}$ are chosen from $\mathbf{P}_{n-1}$ and $\mathbf{Q}_{n}$, they are a possible consecutive pair of vectors in $I$ if and only if

$\mathbf{v}(1) \cdot \mathbf{v}^{\prime}(1)+\mathbf{v}(1) \cdot \mathbf{v}^{\prime}(2 n)=0, \mathbf{v}(i) \cdot \mathbf{v}^{\prime}(2 i-2)=0$ and $\mathbf{v}(i) \cdot \mathbf{v}^{\prime}(2 i-1)=0$ for $i=2,3, \ldots, n$. $I$ can be obtained by the procedure described below. First, take a vector from $\mathbf{Q}_{n}$ such that it corresponds to the first row of $I$, and denote it as $\mathbf{u}$. Second, glue a vector $\mathbf{v}$ from $\mathbf{P}_{n-1}$ to the bottom of $\mathbf{u}$, ensuring that $\mathbf{u}$ and $\mathbf{v}$ are legit consecutive vectors in $I$. Third, glue a vector 
$\mathbf{v}^{\prime}$ from $\mathbf{P}_{n-1}$ to the bottom of $\mathbf{v}$ such that $\mathbf{v}$ and $\mathbf{v}^{\prime}$ are orthogonal. Then we glue $\mathbf{u}^{\prime}$ to the bottom of $\mathbf{v}^{\prime}$, making sure that $\mathbf{v}^{\prime}$ and $\mathbf{u}^{\prime}$ are possible consecutive vectors in $I$. Repeat the above procedure until the $(3 m+1)$-th column is glued.

Consider the transfer matrix of $H_{m, n}$. The transfer matrix $B_{n_{1}}$, which represents every $(3 k+1)$-th $(0 \leq k \leq n-1)$ step, can be defined as a $3^{n} \times 2^{n}$ matrix of 0 's and 1's as follows. The rows of $B_{n_{1}}$ are indexed by vectors of $\mathbf{Q}_{n}$ and columns are indexed by vectors of $\mathbf{P}_{n-1}$, and the entry of $B_{n_{1}}$ in position $(\alpha, \beta)$ is 1 if $\alpha, \beta$ represent possible consecutive pair of rows in an independent set of $H_{m, n}$, and is 0 otherwise. Let $B_{n_{3}}$ denote the transfer matrix that represents every $(3 k+3)$-th $(0 \leq k \leq n-1)$ step. It is no difficult to see that $B_{n_{3}}$ is the transpose of $B_{n_{1}}$. The transfer matrix $B_{n_{2}}$ that represents every $(3 k+2)$-th $(1 \leq k \leq n-1)$ step is a $2^{n} \times 2^{n}$ matrix whose rows and columns are indexed by vectors of $\mathbf{P}_{n-1}$. The entry of $B_{n_{2}}$ in position $(\alpha, \beta)$ is 1 if $\alpha, \beta$ are orthogonal, and is 0 otherwise. Thus if we take the transfer matrix of $H_{m, n}$ to be $B_{n}=B_{n_{1}} B_{n_{2}} B_{n_{3}}$, for the Merrified-Simmons index of $H_{m, n}$, we have

$$
g(m, n)=\mathbf{1} \cdot B_{n}^{m} \mathbf{1},
$$

where 1 denotes the all one column vector.

When $n=2$, the transfer matrices $B_{2_{1}}, B_{2_{2}}$ and $B_{2_{3}}$ are

$$
B_{2_{1}}=\left(\begin{array}{cccc}
1 & 1 & 1 & 1 \\
1 & 1 & 0 & 0 \\
1 & 0 & 1 & 0 \\
1 & 0 & 1 & 0 \\
1 & 0 & 0 & 0 \\
1 & 0 & 1 & 0 \\
1 & 1 & 0 & 0 \\
1 & 1 & 0 & 0 \\
1 & 0 & 0 & 0
\end{array}\right), B_{2_{2}}=\left(\begin{array}{cccc}
1 & 1 & 1 & 1 \\
1 & 0 & 1 & 0 \\
1 & 1 & 0 & 0 \\
1 & 0 & 0 & 0
\end{array}\right), B_{2_{3}}=B_{2_{1}}^{\top}
$$

Therefore, the triple-step transfer matrix of $H_{m, n}$ is

$$
B_{2}=\left(\begin{array}{lllllllll}
9 & 6 & 6 & 6 & 4 & 6 & 6 & 6 & 4 \\
6 & 3 & 4 & 4 & 2 & 4 & 3 & 3 & 2 \\
6 & 4 & 3 & 3 & 2 & 3 & 4 & 4 & 2 \\
6 & 4 & 3 & 3 & 2 & 3 & 4 & 4 & 2 \\
4 & 2 & 2 & 2 & 1 & 2 & 2 & 2 & 1 \\
6 & 4 & 3 & 3 & 2 & 3 & 4 & 4 & 2 \\
6 & 3 & 4 & 4 & 2 & 4 & 3 & 3 & 2 \\
6 & 3 & 4 & 4 & 2 & 4 & 3 & 3 & 2 \\
4 & 2 & 2 & 2 & 1 & 2 & 2 & 2 & 1
\end{array}\right)
$$

The 4-8-8 lattice with toroidal boundary condition, denote as $T_{m, n}$, can be obtained by identifying edges $\left(b_{i}, b_{i+1}\right)$ with $\left(b_{i}^{*}, b_{i+1}^{*}\right)(1 \leq i \leq 2 n-1)$ of $H_{m, n}$ respectively (see Fig.3).

In general, for a lattice $P_{m, n}$ with free boundary condition and transfer matrix $T_{m}$, let us denote its corresponding lattices with cylindrical boundary condition and toroidal boundary condition by $C_{m, n}$ (with transfer matrix $B_{n}$ ) and $T_{m, n}$ respectively. 
We say that the square lattice is of transfer multiplicity one since computing the MerrifieldSimmons index for it only needs to employ single-step transfer matrices as shown in [5]. This may not hold for other lattices. For example, in [23], for a generalized aztec diamond we need to introduce double-step transfer matrices, therefore the transfer multiplicity of a generalized aztec diamond is two. Based on the discussion above, the 4-8-8 lattice is of transfer multiplicity three. The triangular kagomé lattice has multiplicity four since quadruple-step transfer matrices need to be involved in computing its Merrifield-Simmons index. The entropy constant of a lattice with free boundary condition is defined by $\eta=\lim _{m, n \rightarrow \infty} f(m, n)^{1 / k(m, n)}$ where $f(m, n)$ is the Merrifield-Simmons index of $P_{m, n}$ and $k(m, n)$ denotes the number of vertices of $P_{m, n}$. The entropy of the lattices with cylindrical or toroidal boundary conditions can be defined similarly. As in [5], we have that the Merrifield-Simmons index for $P_{m, n}, C_{m, n}$ and $T_{m, n}$ are $1 \cdot T_{m}^{n} 1,1 \cdot B_{n}^{m} 1$ and $\operatorname{Trace}\left(1 \cdot B_{n}^{m} 1\right)$ respectively.

Lemma 2.2. Let $P_{m, n}$, whose transfer matrix is denoted by $T_{m}$, be a lattice with free boundary condition for given positive integers $m$ and $n$. Let $C_{m, n}$, whose transfer matrix is denoted by $B_{n}$, be the corresponding lattice with cylindrical boundary condition and $T_{m, n}$ be the corresponding lattice with toroidal boundary condition. Then the trace of $T_{m}^{n}$ is equal to the Merrifield-Simmons index of $C_{m, n}$ and the trace of $B_{n}^{m}$ is equal to the Merrifield-Simmons index of $T_{m, n}$.

Proof. Recall that $C_{m, n}$ can be obtained by identifying the leftmost and the rightmost column of $P_{m, n}$. Thus there is a bijection between the independent sets of $C_{m, n}$ and the independent sets of $P_{m, n}$ whose leftmost and rightmost column vectors are the same. And the latter is the trace of $T_{m}^{n}$.

Similarly, $T_{m, n}$ can be obtained by identifying the top row and the bottom row of $C_{m, n}$. Thus there is a bijection between the independent sets of $T_{m, n}$ and the independent sets of $C_{m, n}$ whose corresponding top and bottom row vectors are the same. And the latter is the trace of $B_{n}^{m}$.

In [5], Calkin and Wilf proved the existence of the entropy constant of the square lattice and established its upper and lower bounds. Note that the method provided by Calkin and Wilf is valid for the lattices with the same symmetric transfer matrices in both horizontal and vertical directions. Thus, the bounds can be generalized and stated as follows.

Theorem 2.3. Let $P_{m, n}$ be a lattice with the same real symmetric transfer matrix $T_{m}$ in both horizontal and vertical directions. Let $B_{n}$ be the transfer matrix of the corresponding lattice with cylindrical boundary condition. For both lattices, the entropy constant of the Merrifield-Simmons index is lower bounded by $\left(\frac{\lambda_{p+2 q}}{\lambda_{2 q}}\right)^{1 / p}$ where $\lambda$ 's are the largest eigenvalues of corresponding $T$ 's. And the upper bound of the entropy constant is $\left(\xi_{2 k}\right)^{1 / 2 k}$ where $\xi$ 's are the largest eigenvalues of corresponding $B$ 's. 


\section{Numerical results}

Note that the 4-8-8 lattice has the same symmetric transfer matrix in both horizontal and vertical directions. By Lemma 2.2, we can apply Theorem 2.3 to derive the lower and upper bound of entropy constant for the 4-8-8 lattice.

Let $p=2, q=3$ and $k=4$ in Theorem 2.3, since the largest eigenvalues of $T_{6}, T_{8}$ and $B_{8}$ are $105606.367915106937 \ldots, 3510407.307349548675 \ldots$ and $1220870.544468111359 \ldots$ respectively. We have

$$
5.765456527051 \ldots \leq \lim _{m, n \rightarrow \infty} f(m, n)^{1 / m n} \leq 5.765456529341 \ldots,
$$

where $f(m, n)$ is the Merrifield-Simmons index of the 4-8-8 lattice $G_{m, n}$. Since the number of vertices of $G_{m, n}$ is $4 m n+2 m+2 n$, we can see that the entropy constant of the 4-8-8 lattice is

$$
\eta=\lim _{m, n \rightarrow \infty} f(m, n)^{1 / 4 m n+2 m+2 n},
$$

which is between $1.549560101247 \ldots$ and $1.549560101400 \ldots$.

Some numerical results of Merrifield-Simmons index of the 4-8-8 lattice with various boundary conditions are presented in the following tables.

Table 1: The numerical results of Merrifield-Simmons index of the 4-8-8 lattice $G_{m, n}$ with free

\begin{tabular}{|c|c|c|c|c|}
\hline$n$ & $f(1, n)$ & $f(2, n)$ & $f(3, n)$ & $f(4, n)$ \\
\hline 1 & 47 & 779 & 12887 & 213203 \\
\hline 2 & 779 & 74753 & 7144259 & 682899449 \\
\hline 3 & 12887 & 7144259 & 3935795807 & 2168825644779 \\
\hline 4 & 213203 & 682899449 & 2168825644779 & 6890559309027361 \\
\hline 5 & 3527231 & 65275954619 & 1195119855466343 & 21891571255589181023 \\
\hline 6 & 58354523 & 6239500931057 & 658564832936254227 & 69550417915510873198592 \\
\hline 7 & 965417447 & 596412129451379 & 362898854276036750752 & 220964515515328798010638336 \\
\hline 8 & 15971869859 & 57008955129453545 & 199973597225797700796416 & 702013283095176099770505625600 \\
\hline 9 & 264238674767 & 5449287169347507444 & 110194449810190513907695616 & \\
\hline$n$ & $f(5, n)$ & $f(6, n)$ & $f(7, n)$ & $f(8, n)$ \\
\hline 1 & 3527231 & 58354523 & 965417447 & 15971869859 \\
\hline 2 & 65275954619 & 6239500931057 & 596412129451379 & 57008955129453545 \\
\hline 3 & 1195119855466343 & 658564832936254227 & 362898854276036750752 & 199973597225797700796416 \\
\hline 4 & 21891571255589181059 & 69550417915510873198592 & 220964515515328798010638336 & 702013283095176099770505625600 \\
\hline 5 & 400989410760263959456896 & 7344958142436702598532694016 & & \\
\hline 6 & 7344958142436702598532694016 & & & \\
\hline$n$ & $f(9, n)$ & & & \\
\hline 1 & 264238674767 & & & \\
\hline 2 & 5449287169347507444 & & & \\
\hline 3 & 110194449810190513907695616 & & & \\
\hline
\end{tabular}
boundary condition

\section{Final remark}

1. This transfer matrix method for computing Merrifield-Simmons index is also valid for non-planar lattices. For instance, the square lattice with crossed diagonal bonds (obtained by adding two crossed diagonals to each square inner face) is non-planar; its Merrifield-Simmons index can be computed by using multi-step transfer matrix and it can be showed that the entropy constant is between $1.342542258 \ldots$ and $1.342652572 \ldots$

2. The 4-8-8 lattice has larger entropy constant of Merrifield-Simmons index comparing to that of the square lattice [5] while the square lattice has larger entropy than that of the square 
Table 2: The numerical results of Merrifield-Simmons index of the 4-8-8 lattice $H_{m, n}$ with cylindrical boundary condition

\begin{tabular}{|c|c|c|c|c|}
\hline$m$ & $g(m, 1)$ & $g(m, 2)$ & $g(m, 3)$ & $g(m, 4)$ \\
\hline 1 & 15 & 275 & 4527 & 74915 \\
\hline 2 & 81 & 9223 & 872817 & 83484359 \\
\hline 3 & 435 & 308007 & 167161419 & 92210259455 \\
\hline 4 & 2337 & 10288055 & 32024704905 & 101890687050895 \\
\hline 5 & 12555 & 343638751 & 6135187215867 & 112585183506906592 \\
\hline 6 & 67449 & 11478130575 & 1175359722363369 & 124402293749613166592 \\
\hline 7 & 362355 & 383389471367 & 225171683166443808 & 137459739741954729050112 \\
\hline 8 & 1946673 & 12805873388263 & 43137676077348913152 & 151887714595812392365457408 \\
\hline 9 & 10458075 & 427738384795215 & 8264179008000619446272 & 167830070726599585943198367744 \\
\hline$m$ & $g(m, 5)$ & $g(m, 6)$ & $g(m, 7)$ & $g(m, 8)$ \\
\hline 1 & 1239375 & 20504243 & 339222255 & 5612094275 \\
\hline 2 & 7979610641 & 762745733191 & 72908185459505 & 6969039275744391 \\
\hline 3 & 50808099454115 & 27997712914113532 & 15427992474894299136 & 8501518342886229278720 \\
\hline 4 & 323676087966247552 & 1028340702944578895872 & 3267078212691260344369152 & 10379641364400127593513222144 \\
\hline 5 & 2061947765452025364480 & 37769283626796604821864448 & 691822641713709142974429921280 & \\
\hline 6 & 13135457408094420540063744 & 1387206347469407026513191108608 & & \\
\hline 7 & 83678274939057768517536317440 & & & \\
\hline
\end{tabular}

Table 3: The numerical results of Merrifield-Simmons index of the 4-8-8 lattice $S_{m, n}$ with toroidal boundary condition

\begin{tabular}{|c|c|c|c|c|}
\hline$m$ & $s(m, 1)$ & $s(m, 2)$ & $s(m, 3)$ & $s(m, 4)$ \\
\hline 1 & 5 & 29 & 155 & 833 \\
\hline 2 & 29 & 1127 & 37235 & 1244819 \\
\hline 3 & 155 & 37235 & 7023713 & 1347121127 \\
\hline 4 & 833 & 1244819 & 1347121127 & 1490864952223 \\
\hline 5 & 4475 & 41575979 & 258054952385 & 1647144129284543 \\
\hline 6 & 24041 & 1388718947 & 49437673996703 & 1820049164089801216 \\
\hline 7 & 129155 & 46385594795 & 9471107791983884 & 2011082476723567853568 \\
\hline 8 & 693857 & 1549359408899 & 1814444828776562944 & 2222168789066396131655680 \\
\hline 9 & 3727595 & 51751291603979 & 347605577830867337216 & 2455410851801241684155564032 \\
\hline$m$ & $s(m, 5)$ & $s(m, 6)$ & $s(m, 7)$ & $s(m, 8)$ \\
\hline 1 & 4475 & 24041 & 129155 & 693857 \\
\hline 2 & 41575979 & 1388718947 & 46385594795 & 1549359408899 \\
\hline 3 & 258054952385 & 49437673996703 & 9471107791983884 & 1814444828776548608 \\
\hline 4 & 1647144129284543 & 1820049164089801216 & 2011082476723567853568 & 2222168789066506995499008 \\
\hline 5 & 10491381016588933120 & 66835240986806694445056 & 425767589675573485997391872 & 2712315179113658136571668332544 \\
\hline 6 & 66835240986806694445056 & 2454789445716724048587653120 & 90160437291592209800637701947392 & \\
\hline 7 & 425767589675573485997391872 & 90160437291592209800637701947392 & & \\
\hline 8 & 712315179113658136571668332544 & & & \\
\hline
\end{tabular}

lattice with crossed diagonal bonds. Note that the vertices of the 4-8-8 lattice are of degree three while the vertices of the square lattice and square lattice with crossed diagonal bonds are of degree four and eight respectively. Is the entropy constant of Merrifield-Simmons index negatively correlated to the average degree of a lattice? Intuitively, the answer is likely to be a yes but further investigation needs to be made.

3. By straightforward combinatorial arguments, we can see that for Merrifield-Simmons index, the entropy constants of lattices with free boundary condition are the same as the entropy constants of the corresponding cylindrical and toroidal lattices. But this phenomenon may disappear for some other models. For instance, the the boundary condition of a honeycomb lattice has a significant effect on its entropy constant in dimer problem, see [10,11,21].

\section{Acknowledgments}

I would like to thank Zhibo Chen and Hong-Jian Lai for their helpful comments.

\section{References}


[1] G. R. Allen, Dimer models for the antiferroelectric transition in copper formate tetrahydrate, Journal of Chem. Phys. 60 (1974) 3299-3309.

[2] R. J. Baxter, Exactly Solved Models in Statistical Mechanics, Academic Press, London, 1982.

[3] R. J. Baxter, Planar Lattice Gas with nearest-Neighbor Exclusion, J. Annals of Combin. 3(1999) 191-203.

[4] R. J. Baxter, I. G. Enting, and S. K. Tsang, Hard-Square Lattice Gas, J. Statist. Phys. 22 (1980) 465-489.

[5] N. J. Calkin and H. S. Wilf, The number of independent sets in a grid graph, SIAM J. Discrete Math. 11 (1998) 54-60.

[6] C. Domb and M. S. Green (Eds.), Phase Transitions and Critical Phenomena, Vol. 1, Academic Press, London, 1972.

[7] S. R. Finch, Several constants arising in statistical mechanics, Journal Annals of Combinatorics 3 (1999) 323-335.

[8] I. Gutman, Extremal hexagonal chains, J. Math. Chem. 12 (1993) 197-210.

[9] A. Haji-Ankabari and R.M. Ziff, Bond percolation on the triangular kagomé lattice, Phys. Rev. E 79 (2009) 021118-021125.

[10] D. J. Klein, G. E. Hite, and T. G. Schmalz, Transfer-matrix method for subgraph enumeration: Application to polypyrene fusenes, J. Comput. Chem. 7 (1986) 443-456.

[11] D. J. Klein and T.G. Schmalz, Exact enumeration of long-range-ordered dimer coverings on the square-planar lattice, Phys. Rev. B 41 (1990) 2244-2248.

[12] X. Liu and W. G. Yan, The triangular kagomé lattices revisited, Physica A 392(22) 56155621.

[13] Y. L. Loh, D. X. Yao and C. L. Carlson, Closed-packed dimers on the triangular kagomé lattice, Phys. Rev. B 78 (2008) 224410-224419.

[14] S.Maruti and L. W. ter Haar, Magnetic properties of the two-dimensional "triangles-intriangles" Kagomé lattice $\mathrm{Cu}_{9} \mathrm{X}_{2}(\mathrm{cpa})_{6}$ (X=F, CI, Br), J. Appl. Phys. 75 (1993) 59495951.

[15] R. E. Merrifield and H. E. Simmons, Topological Methods in Chemistry, Wiley, New York, 1989.

[16] P. A. Pearce and K. A. Seaton, A Classical Theory of Hard Squares, J. Statist. Phys. 53 (1998) 1061-1072.

[17] H. Ren and F. J. Zhang, Double hexagonal chains with maximal Hosoya index and minimal Merrifield-Simmons index, J. Math. Chem. 42 (2007) 679-690.

[18] S. R. Salinas and J. F. Nagle, Theory of the phase transition in the layered hydrogenbonded $\mathrm{SnCL}^{2} \cdot 2 \mathrm{H}_{2} \mathrm{O}$ crystal, Phys. Rev. B 9(1974) 4920-4931. 
[19] R. Shrock and F. Y. Wu, Spanning trees on graphs and lattices in d dimensions, J. Phys. A: Math. Gen. 33 (2000) 3881-3902.

[20] F. Y. Wu, Dimers on two-dimensional lattices, Int. J. Mod. Phys. B 20(2006) 5357-5371.

[21] W. G. Yan, Y.-N. Yeh, and F. J. Zhang, Dimer problem on the cylinder and torus, Physica A 387 (2008) 6069-6078.

[22] L. Zhang, On the ordering of a class of hexagonal chain with respective to Merrifield Simmons index, Systems Sci. Math. Sci. 13 (2000) 219-224.

[23] Z. Zhang, Merrifield-Simmons Index of Generalized Aztec Diamond and Related Graphs, MATCH communications in mathematical and in computer chemistry 56 (2006) 625-636.

[24] L. Zhang and F. Tian, Extremal catacondensed benzenoids, J. Math. Chem. 34(2003) 111-122.

[25] Y. Zeng and F. J. Zhang, Extremal polyomino chains on $k$-matchings and $k$-independent sets, J. Math. Chem. 42 (2007) 125-140. 


\section{University Library}

\section{- M M I E E R VA A gateway to Melbourne's research publications}

Minerva Access is the Institutional Repository of The University of Melbourne

Author/s:

Zhang, Z

Title:

Merrifield-Simmons Index and Its Entropy of the 4-8-8 Lattice

Date:

2014-02-01

Citation:

Zhang, Z. (2014). Merrifield-Simmons Index and Its Entropy of the 4-8-8 Lattice. Journal of Statistical Physics, 154 (4), pp.1113-1123. https://doi.org/10.1007/s10955-013-0883-9.

Persistent Link:

http://hdl.handle.net/11343/282907 